\title{
Correction to: Does Education Make People Happy? Spotlighting the Overlooked Societal Condition
}

\section{Satoshi Araki ${ }^{1}$}

Published online: 16 October 2021

(c) Springer Nature B.V. 2021

\section{Correction to: Journal of Happiness Studies} https://doi.org/10.1007/s10902-021-00416-y

In the original publication, Table 11 has been published incorrectly. The correct version is given in this correction.

The original article has been corrected.

The original article can be found online at https://doi.org/10.1007/s10902-021-00416-y.

Satoshi Araki

satoshiaraki@ln.edu.hk

1 Department of Sociology and Social Policy, Lingnan University, 8 Castle Peak Road, Tuen Mun, New Territories, Hong Kong 
Table 11 Multilevel linear regression of life satisfaction (with occupations and income included separately)

\begin{tabular}{|c|c|c|c|c|}
\hline \multirow[t]{2}{*}{ Predictor variables } & \multicolumn{2}{|l|}{ Model A6-1 } & \multicolumn{2}{|l|}{ Model A6-2 } \\
\hline & $\mathrm{B}$ & S.E. & $\mathrm{B}$ & S.E. \\
\hline Intercept & $4.067 * * *$ & 0.407 & $3.284 * * *$ & 0.272 \\
\hline \multicolumn{5}{|l|}{ Level one (individual) } \\
\hline Tertiary education & $0.764 * *$ & 0.233 & 0.308 & 0.178 \\
\hline Age & $-0.079 * * *$ & 0.007 & $-0.069 * * *$ & 0.006 \\
\hline Age squared & $0.001 * * *$ & 0.000 & $0.001 * * *$ & 0.000 \\
\hline Men & $-0.099 * *$ & 0.035 & $-0.131 * * *$ & 0.032 \\
\hline \multicolumn{5}{|l|}{ Marital status (reference $=$ others) } \\
\hline Legally married & $0.490 * * *$ & 0.046 & $0.382 * * *$ & 0.044 \\
\hline Legally registered civil union & $0.433 * * *$ & 0.097 & $0.367 * * *$ & 0.100 \\
\hline Legally separated & $-0.467 * * *$ & 0.089 & $-0.326^{* *}$ & 0.100 \\
\hline Legally divorced/dissolved & $-0.129 *$ & 0.055 & -0.013 & 0.053 \\
\hline Widowed/partner died & -0.029 & 0.092 & 0.040 & 0.088 \\
\hline Living with children & -0.059 & 0.035 & 0.019 & 0.030 \\
\hline Trust & $0.163 * * *$ & 0.007 & $0.136^{* * *}$ & 0.007 \\
\hline Religion or denomination & $0.098^{*}$ & 0.042 & $0.116^{* *}$ & 0.034 \\
\hline \multicolumn{5}{|l|}{ Voluntary/charitable activity (reference $=$ never) } \\
\hline At least once a week & $0.289 * * *$ & 0.052 & $0.248 * * *$ & 0.048 \\
\hline At least once a month & $0.234 * * *$ & 0.047 & $0.175^{* * *}$ & 0.044 \\
\hline At least once every three months & $0.183 * * *$ & 0.049 & $0.112 *$ & 0.045 \\
\hline At least once every six months & 0.137 & 0.078 & 0.077 & 0.073 \\
\hline Less often & 0.040 & 0.044 & 0.015 & 0.040 \\
\hline \multicolumn{5}{|l|}{ Health status (reference $=$ very bad) } \\
\hline Very good & $3.019 * * *$ & 0.142 & $2.510 * * *$ & 0.137 \\
\hline Good & $2.616 * * *$ & 0.121 & $2.160 * * *$ & 0.119 \\
\hline Fair & $1.940 * * *$ & 0.132 & $1.617 * * *$ & 0.135 \\
\hline $\mathrm{Bad}$ & $1.070 * * *$ & 0.146 & $0.960 * * *$ & 0.146 \\
\hline \multicolumn{5}{|l|}{ Main activity (reference $=$ others) } \\
\hline Paid work & 0.014 & 0.045 & & \\
\hline Education & 0.015 & 0.094 & & \\
\hline Unemployed/looking for a job & $-0.927 * * *$ & 0.233 & & \\
\hline Unemployed/not looking for a job & -0.830 & 0.478 & & \\
\hline Permanently sick/disabled & -0.276 & 0.148 & & \\
\hline Retired & 0.036 & 0.062 & & \\
\hline Community/military service & -1.150 & 0.698 & & \\
\hline Housework/looking after others & -0.101 & 0.098 & & \\
\hline \multicolumn{5}{|l|}{ Income (reference $=$ very difficult $)$} \\
\hline Comfortable & & & $2.094 * * *$ & 0.110 \\
\hline Coping & & & $1.613 * * *$ & 0.087 \\
\hline Difficult & & & $0.823 * * *$ & 0.066 \\
\hline \multicolumn{5}{|l|}{ Cross-level interactions } \\
\hline Tertiary education $*$ educational expansion & -0.010 & 0.007 & -0.006 & 0.005 \\
\hline Tertiary education $*$ skills diffusion & $-0.023 * *$ & 0.009 & -0.013 & 0.007 \\
\hline
\end{tabular}


Table 11 (continued)

\begin{tabular}{|c|c|c|c|c|}
\hline \multirow[t]{2}{*}{ Predictor variables } & \multicolumn{2}{|l|}{ Model A6-1 } & \multicolumn{2}{|l|}{ Model A6-2 } \\
\hline & $\mathrm{B}$ & S.E. & $\mathrm{B}$ & S.E. \\
\hline \multicolumn{5}{|l|}{ Level two (country) } \\
\hline Educational expansion & -0.004 & 0.012 & -0.003 & 0.009 \\
\hline Skills diffusion & $0.102 * * *$ & 0.021 & $0.076^{* * *}$ & 0.016 \\
\hline \multicolumn{5}{|l|}{ Variance (random effect) } \\
\hline Covariance structure (intercept) & 0.257 & & 0.132 & \\
\hline Tertiary education & 0.051 & & 0.029 & \\
\hline
\end{tabular}

The analytic model is based on Model 4 in the main manuscript, and individual-level "Main Activity" and "Income" are included separately.

$* * * p<0.001, * * p<0.01, * p<0.05$ [two tailed] [N: Individual $=48,247$, Country $=24$ ]

Publisher's Note Springer Nature remains neutral with regard to jurisdictional claims in published maps and institutional affiliations. 